\title{
Embolotherapy in the Management of Hepatocellular Carcinoma
}

\author{
Alireza Mojtahedi, M.D., ${ }^{1}$ Xiaoming Yang, M.D., Ph.D., ${ }^{1}$ \\ and Gaurav K. Goswami, M.D.'
}

Hepatocellular carcinoma (HCC) ranks fifth in frequency of cancers worldwide. The incidence of HCC in the United States is rising, primarily due to the number of patients who were infected by hepatitis in the 1960s and 1970s coupled with the rising migrant population from Asia, where hepatitis is widely prevalent. Up to $80 \%$ of the patients present with multicentric HCC and advanced liver disease or comorbidities that restrict the option of resection or liver transplantation. The dual blood supply (arterial and portal) to the liver with predominantly arterial supply to the tumor has made embolotherapy a cornerstone in the management of inoperable HCC. The techniques have become refined not only due to the development of microcatheter angiographic capabilities, but also in the ability to deliver a wide variety of therapeutic agents to these tumors. This article reviews the fundamental principles of bland embolization, chemoembolization, and radioembolization in the management of $\mathrm{HCC}$.

KEYWORDS: Embolization, hepatocellular carcinoma, chemoembolization, radioembolization

Objectives: On completion of this article, the reader should understand (1) the role of embolotherapy in management of hepatocellular carcinoma, and (2) the unique features involved with the use of different embolic agents in patients with hepatocellular carcinoma. Accreditation: Tufts University School of Medicine (TUSM) is accredited by the Accreditation Council for Continuing Medical Education to provide continuing medical education for physicians.

Credit: TUSM designates this educational activity for a maximum of 1 AMA PRA Category 1 Credit $^{\mathrm{TM}}$. Physicians should only claim credit commensurate with the extent of their participation in the activity.

The incidence of hepatocellular carcinoma $(\mathrm{HCC})$ is on the increase. With a rise in cases with viral hepatitis and cirrhosis, HCC ranks fifth in frequency of cancers worldwide. Major etiologies of $\mathrm{HCC}$ are chronic viral hepatitis B, C, and D; alcohol; metabolic liver disease such as hemochromatosis and alfa-1-antitrypsin deficiency; autoimmune hepatitis; nonalcoholic fatty liver disease; obesity; and diabetes mellitus. ${ }^{1}$ Both surgical resection and orthotopic liver transplantation offer the best chance for cure and are the only established definitive treatments. Despite the increased use of screening, the majority of patients with HCC are not surgical candidates at the time of their initial presentation due to tumor size, metastasis, or poor hepatic function. Unlike cancers that develop in an otherwise normal organ, HCC develops in a liver already damaged by hepatitis, cirrhosis, or portal hypertension. Patients and their physicians are fighting a battle on two fronts, treating cancer and preserving liver function. Most of the deaths in patients with HCC are due to hepatic failure or to gastrointestinal (GI) bleeding secondary to portal hypertension. ${ }^{1-6}$
${ }^{1}$ Department of Radiology, Section of Interventional Radiology, University of Washington School of Medicine, Seattle, Washington. Address for correspondence and reprint requests: Gaurav $\mathrm{K}$. Goswami, M.D., University of Washington Medical Center, 1959 NE Pacific St, Seattle, WA 98195 (e-mail: goswamig@u.washington.edu).
Embolization 2008; Guest Editor, Thomas R. Burdick, M.D.

Semin Intervent Radiol 2008;25:234-241. Copyright (C) 2008 by Thieme Medical Publishers, Inc., 333 Seventh Avenue, New York, NY 10001, USA. Tel: +1 (212) 584-4662.

DOI 10.1055/s-0028-1085922. ISSN 0739-9529. 
Systemic chemotherapy has not been proved to be effective in management of HCC. ${ }^{1}$ The recent U.S. Food and Drug Administration (FDA) approval of sorafenib (Nexavar) has renewed interest in systemic therapy, and phase III trials with this agent have shown some survival benefits, although further studies are required to define its toxicities and exact role in the management of $\mathrm{HCC}^{7}$ Given the limited therapeutic options in patients with $\mathrm{HCC}$ at the time of presentation, IR has played and continues to play an increasingly important role in their management. By reducing tumor burden, controlling symptoms like abdominal pain, improving overall survival, and, in some cases, providing an effective bridge to transplant or making patients eligible for resection on second look, IR treatments offer a much needed hope against a disease with such poor overall prognosis. Among the spectrum of treatments offered are thermal ablations, a variety of catheter-based embolization techniques, and combination therapies such as ablation before or after embolization. In keeping with the theme of this issue, this article is limited to discussion of embolotherapy in management of nonoperative HCC. Preoperative embolization and portal vein embolization are covered in other articles.

Normal hepatocytes receive blood supply from both the hepatic artery (20 to 30\%) and the portal vein (70 to $80 \%$ ). In contrast, malignant liver tumors are mainly fed by branches of the hepatic artery. Nature's gift of dual (portal and arterial) blood supply to the liver, coupled with the predominantly arterial blood supply to the tumors, lends the opportunity to aggressively offer arterial embolization therapies. These include bland embolization, chemoembolization, and radioembolization, which are all primarily directed toward treatment of the tumors. Arterial embolization primarily involves selective and superselective catheterization of hepatic arteries and their branches and delivery of an agent (Fig. 1). It is effective due to the double impact of inducing tumor ischemia and, in cases where a therapeutic agent (chemotherapeutic drugs or radiation particles) is delivered, localizing a high concentration of the agent within the tumor while limiting systemic toxicity. The embolization works on the principle of vascular flow carrying the embolic particle into the tumor. Hence it is important that the catheterization itself is not too selective, and that during embolization there is enough flow around the catheter so the agent is carried well into the arterioles feeding the tumor. This technique also offers the benefit of somewhat targeted treatment with relative sparing of surrounding liver parenchyma, which is an important consideration due to the commonly limited available hepatic reserve secondary to cirrhosis.

Because eligibility criteria for most forms of embolization and their subsequent postprocedure follow-up care are fairly similar, these aspects of the procedures are discussed under a common heading.
Unique features applicable to each therapeutic agent are discussed separately.

\section{PATIENT SELECTION}

The clinical management in patients with $\mathrm{HCC}$ is challenging because of tumoral involvement in hepatic parenchyma, which is often severely damaged by chronic inflammation and underlying cirrhosis, especially in patients with hepatitis C. Physicians must walk a fine line in treating the tumor and maintaining adequate liver function. Although no absolute criteria exist, the following clinical concepts and laboratory markers may serve as a guideline to minimize risks and avoid fulminant hepatic failure.

\section{Imaging}

It is important to have recent imaging, ideally within the last 3 months. The modality of choice varies by institution. A baseline four-phase liver computed tomography (CT) scan or a contrast-enhanced magnetic resonance imaging (MRI) scan is usually obtained. Classical imaging findings of arterial enhancement and complete washout have become fairly diagnostic and obviate the need for biopsy in most patients. ${ }^{3}$ When radioembolization is being considered, a positron emission tomography (PET) scan is preferred, although few patients with $\mathrm{HCC}$ are PET avid. Establishing the absence of extrahepatic disease is important before offering liver-directed therapy. A baseline chest CT should be obtained to rule out metastases.

\section{Tumor Size}

The amount of tumor volume replacing normal liver parenchyma should not exceed $75 \%$. Although this is not an absolute contraindication, embolization of larger tumors certainly increases the risk of liver failure due to an inadequate reserve of uninvolved parenchyma.

\section{Portal Vein Involvement}

A thrombosed portal vein either due to tumor extension (tumor thrombus) or to ordinary clots (bland thrombus), although not an absolute contraindication, does put the patient in a high-risk category. It is recommended that a highly selective embolization be considered in these patients to limit any ischemic damage to noninvolved liver.

\section{Medical Condition}

General health and nutritional status of the patient are important and generally reflect the functional capacity of the liver. Caution is recommended when 


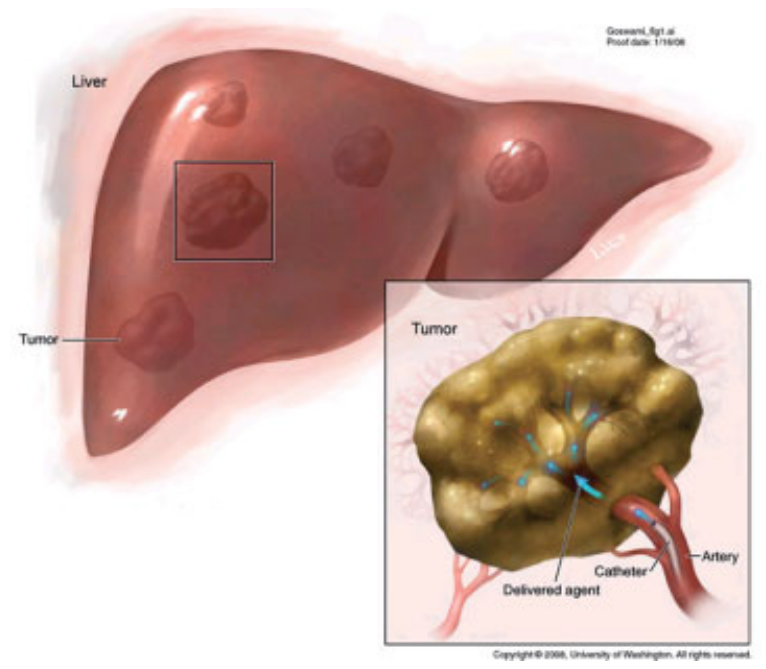

Figure 1 Demonstration of the concept of hepatic artery embolization. A variety of delivery agents can be used, either alone or in combination, and are delivered through a selectively placed intraarterial catheter.

using doxorubicin in elderly patients and in those with decreased cardiac capacity. A left ventricle ejection fraction (LVEF) should be assessed in patients with history of significant cardiac disease. Depending on the severity of cardiac function, the doxorubicin dose can be adjusted or an alternative agents such as cisplatin can be selected. It is important to determine the performance status of the patient. Although minimally invasive, embolization procedures are associated with a fair number of complications. At least an Eastern Cooperative Oncology Group (ECOG) score of 2 or a Karnofsky score of $60 \%$ is required for patients to be able to tolerate these treatments.

\section{Laboratory Measures of Liver Function}

We recommend liver functions tests and cell counts within last 10 days of the procedure, with the following results: lactate dehydrogenase $(\mathrm{LDH})<425 \mathrm{U} / \mathrm{L}$, serum bilirubin $<2.0 \mathrm{mg} / \mathrm{dL}$, platelet count $>50 \times 10^{3} / \mu \mathrm{L}$, neutrophils $>1500 \mathrm{mg} / \mu \mathrm{L}$. Serum glutamic-oxaloacetic transaminase (SGOT) and serum glutamic-pyruvic transaminase (SGPT) are helpful but often tend to be high in most patients secondary to underlying hepatitis. The same is true for $\alpha$-fetoprotein, which is inconsistent as a tumor marker. If the patient develops any new symptoms or there is any change in the clinical status since the patient was scheduled, new laboratory tests should be performed on the day of the procedure.

\section{Suitability of Angiography}

Early on as part of the IR clinic visit, angiographic appropriateness must be assessed. Renal function and the potential for and risks of contrast nephrotoxicity are determined. An assessment of the status of the catheter access site and hepatic arterial anatomy can often be made from the diagnostic CT.

\section{BLAND EMBOLIZATION}

Transcatheter bland embolization has been used as a treatment option for embolization of $\mathrm{HCC}^{8}$ purely to induce tumor ischemia by disrupting the arterial blood supply to the tumor. The technique involves a thorough abdominal and mesenteric angiography to define the blood supply to the tumor(s), followed by selective catheterization and deposition of particles without any added therapeutic drug or agent. The size of particles used varies from 100 to $900 \mu \mathrm{m}$, although most authors use particles ranging in size from 300 to $500 \mu \mathrm{m}$. Fatal pulmonary complications with extremely small size particles have been reported. ${ }^{9}$ Commonly used particulate agents include polyvinyl alcohol (PVA) (Contour, Boston Scientific, Boston, MA), tris-acryl gelatin microspheres (Embosphere, Guerbet Biomedical, Louvres, France), and gelatin sponges (Gelfoam, Upjohn, Kalamazoo, MI). Besides targeting the tumor, bland embolization is also effective for embolization of intratumoral arterioportal and arteriovenous shunts. ${ }^{10}$ When dominant shunts are identified, bland embolization before delivery of chemotherapy or radiation particles may be considered. Some authors believe that bland embolization may be as equally effective as transarterial chemoembolization (TACE) for palliative treatment of primary liver cancer. Despite a trend toward improved survival with TACE, no study to date has demonstrated a difference in survival between the two techniques. ${ }^{11}$ However, no study has documented an improved safety profile with bland embolization when compared with TACE. Protracted postembolization syndrome, liver abscess, and hepatic decompensation have all been reported following bland hepatic artery embolization and TACE.

\section{CHEMOEMBOLIZATION}

Among the various embolotherapies for treatment of hepatic malignancy, TACE has become the most widely used. $^{12-14}$ Selectively delivering chemotherapeutic agents and embolization materials provides high local drug concentration and promotes prolonged retention of the drug in the tumor. Transarterial chemoembolization not only enhances therapeutic effect, it also minimizes systemic toxicities associated with chemotherapy. ${ }^{15,16}$ Many reports have demonstrated the ability of TACE to shrink tumors and delay progression of the disease for many types of hepatic malignancies. The procedure uses the hepatic artery to direct delivery of chemotherapeutic agents from the proximal vessels to the downstream 
small branches that directly supply the lesion. In addition, occlusion of the vessels supplying the mass prevents blood from reaching the tumor, depriving it of oxygen and nutrients and inducing cell necrosis. Generally speaking, normal hepatocytes do not suffer substantial compromise by the TACE procedure because they are able to withstand the ischemic arterial insult due to intact portal vein flow. In addition to the potency of chemotherapeutic agents, the overall therapeutic effect of TACE also depends on the physiological characteristics of the target, including vascularity of the lesions and permeability of the cells. Hypervascular lesions capture more drugs than hypovascular lesions. Furthermore, the tissue hypoxia induced by chemoembolization interrupts the function of $\mathrm{P}$-glycoprotein, an adenosine triphosphate (ATP)-dependent pump ${ }^{15}$ and one of the major mechanisms for drug resistance in liver tumors. Disabling this pump allows for increased drug retention within the cells to achieve maximal antitumor effect.

The protocols for performing TACE vary widely by institutional and individual preferences. Some programs use only single drugs, typically doxorubicin or cisplatin. Others use a combination of three agents: doxorubicin, mitomycin $\mathrm{C}$, and cisplatin. Before infusion into the target vessels, these drugs are usually emulsified with an oil-based radio-opaque agent such as Ethiodol to form a chemoemulsion. Ethiodol (Savage Laboratories, Melville, NY) is selectively retained by HCC cells, serving as a vector for chemotherapeutic agents. This combination helps to increase the dwelltime of the chemotherapy and slows the arterial flow, providing an embolic effect. In addition, some practitioners use permanent embolic agents such as PVA, Embosphere, or Gelfoam in conjunction with chemoemulsion. How these particles are delivered also varies widely in reported trials. Some mix the particles with the chemotherapeutic agent, others deliver particles after the agent has been delivered, and some give particles before and after the chemotherapeutic agent has been delivered. Such wide variations in the technical aspects of performing hepatic artery chemoembolization make evaluation and comparison of TACE literature difficult. There are no consensus data supporting technical superiority of any one method.

Transarterial chemoembolization protocols usually involve several treatment sessions, at intervals of $\sim 4$ weeks. To reduce the likelihood of significant hepatic toxicity, TACE should be restricted to a single lobe or major branch of the hepatic artery at any given session, and repeated once liver toxicities and abnormal chemistries have resolved before continuing the procedure in the remaining segments or lobe. ${ }^{15}$ In patients with portal vein thrombus, elevated bilirubin, and Child-Pugh category of $\mathrm{B}$ or $\mathrm{C}$, a highly selective embolization technique should be employed. Postprocedure laboratory studies and non-contrast-enhanced abdominal CT are obtained after the TACE procedure. This helps to plan future rounds of chemoembolization and evaluate for nontarget embolization (Fig. 2).

The risks of TACE can be avoided or reduced with careful patient selection with regard to liver function, employing careful angiographic techniques and comprehensive postprocedural follow-up. The most common complication of TACE is postembolization syndrome, which includes a wide spectrum of signs and symptoms and, to a variable extent, is seen in almost all patients. It manifests as abdominal pain, nausea, vomiting, transient fever, and malaise. Laboratory tests may show significant rise of serum transaminase (up to 100 -fold), and a leukemoid reaction can occur. ${ }^{15}$ Even
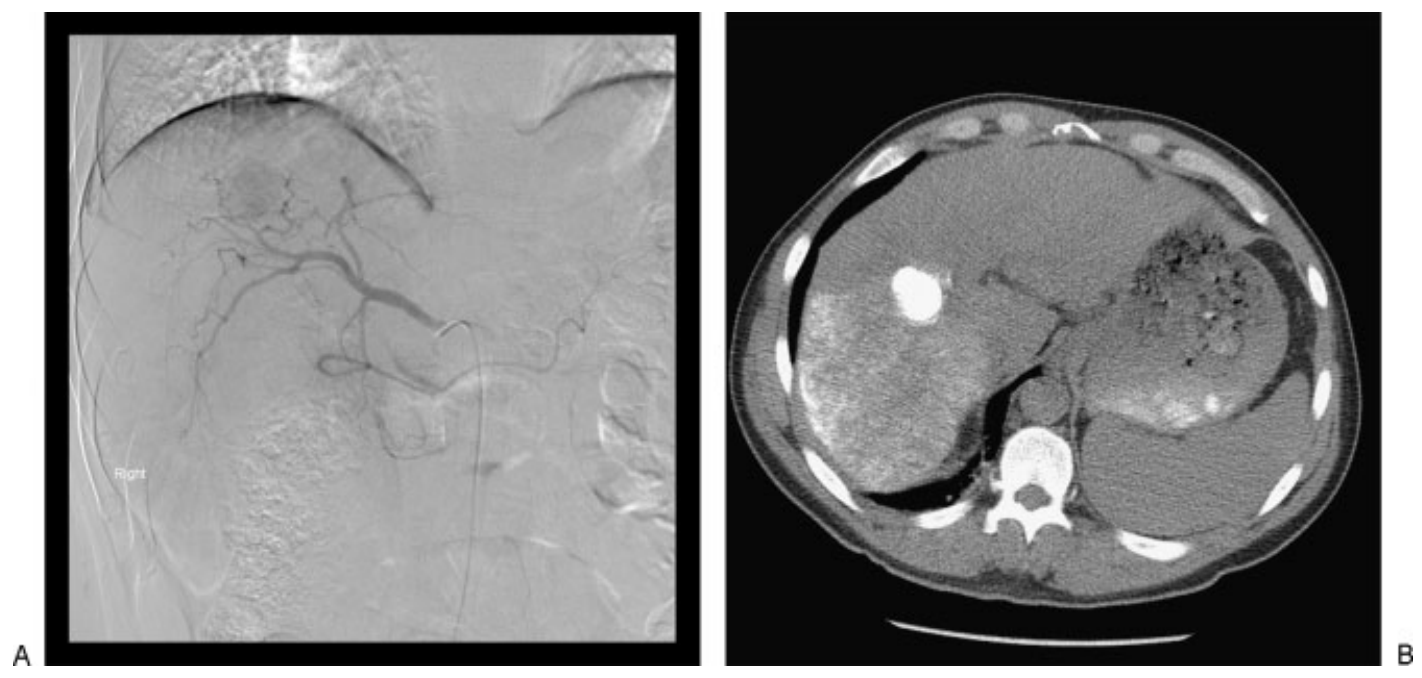

Figure 2 (A) Digital subtraction common hepatic artery angiogram demonstrates a focal hypervascular lesion consistent with hepatocellular carcinoma in 52-year-old man with hepatitis C. (B) Noncontrast computed tomography scan of abdomen obtained after hepatic artery chemoembolization in the same patient shows focal intense uptake of Ethiodol and chemoembolic mixture. Note high density seen in stomach is secondary to ingested material and does not represent nontarget embolization. 
though the delivery of the agent is into the liver, systemic effects of the drugs such as alopecia, pancytopenia, and cardiac arrhythmias have been reported. More serious complications may also arise after the TACE procedure including hepatic insufficiency or infarction, hepatic abscess, biliary necrosis or stricture, tumor rupture, and nontarget embolization to other organs and systems such as the GI tract and gallbladder. The TACE procedurerelated mortality rate is reportedly 2 to $4 \%$.

Transarterial chemoembolization has been proved to delay tumor progression and achieve partial response in 15 to $55 \%$ of patients. ${ }^{10}$ In a preliminary evaluation of our data at the University of Washington, 100 patients with unresectable HCC underwent $~ 324$ hepatic artery chemoembolization procedures over a period of 3 years with doxorubicin + Ethiodol chemoemulsion. Approximately two thirds of patients showed stable disease to partial response lasting up to 1 year. Some of these patients required additional therapy in the form of additional TACE with triple drugs (doxorubicin, cisplatin, and mitomycin), radiofrequency ablation (RFA), or systemic chemotherapy. In the remaining third of $\mathrm{pa}^{-}$ tients, following chemoembolization no response was noted with continued disease progression. Complications were minimal; specifically, there was no case of liver abscess formation.

\section{DRUG ELUTING BEAD EMBOLIZATION}

Although standard chemoembolization selectively delivers drugs into the liver, systemic toxicity can occur and the dwell time of the drug within the tumor may be limited. DC Bead (Biocompatibles, Farnham, Surrey, UK) microspheres, a new embolic material for TACE, have embolization particles that are made from a unique drug-eluting bead (DEB) technology based on a PVA particle containing hydrogel. ${ }^{17}$ These beads allow slow elution of chemotherapy agents. They are available in a variety of sizes and are mixed into a chemotherapeutic agent that leaches the drug into the bead before it is delivered. These drug-loaded carriers are able to deliver drugs in a precise, controlled, and sustained manner to achieve high intratumoral concentration while decreasing systemic release. ${ }^{18}$ Various types of chemotherapeutic agents, commonly doxorubicin and irinotecan, have been be loaded on DEB and are currently being used. Phase 2 trials have shown that high doses of doxorubicin, up to $150 \mathrm{mg}$, can be used without significant systemic toxicity. ${ }^{19}$ Initial studies have shown promising initial results; however, their adverse effects and impact on tumor control and survival are yet to be determined. ${ }^{20}$

\section{RADIOEMBOLIZATION}

Radioembolization is a relatively new treatment that uses catheter-directed selective delivery of radiation particles into hepatic tumors. For years the traditional external beam radiation therapy has had a limited role to play in the treatment of liver tumors because the radiation dose required to kill liver cancer cells far exceeded the dose threshold of surrounding normal liver. Sterotactic radiation therapy has been imprecise given the morphology and location of the tumors within the liver. Several radioactive compounds have been used. Yttrium (Y)-90 is the most common commercially available device. It is produced by bombardment of Y-89 with neutrons in a nuclear reactor. These microspheres range from 20 to $40 \mu \mathrm{m}$ in diameter. Two products (TheraSphere, MDS Nordion, Ottawa, Ontario, Canada; SIR-Spheres, Sirtex Medical, Lane Cove NSW, Australia) are currently being marketed. They differ in the carrier-binding Y-90. In TheraSphere, the Y-90 is a constituent of the glass; in SIR-Spheres, Y-90 is bound to a resin. TheraSphere was approved in 1999 by the FDA under a humanitarian device exemption for the treatment of unresectable HCC. SIR-Spheres received FDA approval in 2002 for the treatment of colorectal metastases to the liver adjunct to intrahepatic floxuridine administration. Yttrium-90 is a pure $\beta$ emitter, with a maximum energy of $2.27 \mathrm{MeV}$ and mean energy of $0.9367 \mathrm{MeV}$. It has a half-life of 2.67 days or 64.2 hours. Once these particles lodge in the arterioles, they impart a very intense local radiotherapeutic effect, penetrating an average area of $2.5 \mathrm{~mm}$, with a maximum penetration of $11 \mathrm{~mm}$ in tissue, before decaying to inactive zirconium-90. It is used in the treatment of both primary and secondary liver tumors. The predominance of arterial (as opposed to portal venous) blood supply to hepatic tumors allows the preferential deposition of microspheres in the tumors rather than the normal parenchyma, thereby maximizing tumor radiation. One gigabecquerel $(\mathrm{GBq})(27 \mathrm{mCi})$ of Y-90 delivers a total absorbed radiation dose of $50 \mathrm{~Gy} / \mathrm{kg}$. Over 11 days, $94 \%$ of the radiation is delivered. ${ }^{21}$

The radiation dose delivered is calculated based on amount of tumor volume, right and left lobe liver volume, and patient body surface area. The following equations have been proposed. ${ }^{22}$

$$
\mathrm{A}(\mathrm{GBq})=\mathrm{D}(\mathrm{Gy}) \times \mathrm{M}(\mathrm{kg}) / 50
$$

where $\mathrm{A}$ is activity, $\mathrm{D}$ is target dose, and $\mathrm{M}$ is the volume of liver to be treated

$$
\mathrm{A}(\mathrm{GBq})=(\mathrm{BSA}-0.2)+\mathrm{TV}(\%) / \mathrm{TLV}(\%)
$$

where $\mathrm{A}$ is activity, BSA is body surface area, TV is tumor volume, and TLV is total liver volume

Patient selection criteria for radioembolization are similar to other techniques of embolization in $\mathrm{HCC}$. The dominant risk is nontarget radiation injury; therefore, these patients undergo a mapping angiogram with prophylactic embolization of gastroduodenal and right gastric arteries. In addition, a systemic 
angiographic search is performed starting with an aortogram with the catheter in supraceliac position. This is followed by the selective superior mesenteric artery (SMA), celiac, common hepatic artery, gastroduodenal artery, right hepatic artery, left hepatic artery, cystic artery, right gastric artery, left gastric artery, phrenic arteries, and any other collaterals that may provide an avenue for Y-90 particles to escape into extrahepatic territories. When identified, these collaterals should be selectively catheterized and coil embolized. The role of power injection angiograms through a microcatheter is extremely important because they lay out the collateral branches that may otherwise be invisible. If considered suitable from an angiographic point of view, at the end of the mapping angiogram technetium methacrylic acid (MAA) particles are injected via the hepatic artery, and the patient undergoes hepatopulmonary shunt evaluation in nuclear medicine. Patients with a hepatopulmonary shunt ratio $>15 \%$ that undergo radioembolization may experience a cumulative dose of more than $30 \mathrm{~Gy}$ to the lungs, increasing the risk for radiation pneumonitis. Some authors propose hepatic arterial bland embolization in an attempt to reduce the shunt and allow safe radioembolization.

On the day of treatment, a selective angiogram of the target lobe of the liver is performed, and continued occlusion of previously performed collaterals is confirmed. New collateral pathways formed in the interim following previous embolization should be excluded. The delivery apparatus is then connected and the particles are delivered. Following delivery of particles a bremsstrahlung scan is obtained (Fig. 3). These gamma ray emissions are produced as a result of interaction of highenergy $\beta$ emission with tissue. This scan helps to show the distribution of microspheres in the liver and may also enable detection of suspected extrahepatic deposition.

Postembolization syndrome following radioembolization is significantly less intense from that experienced with other embolic therapies. It is fairly well tolerated, with some patients reporting vague abdominal
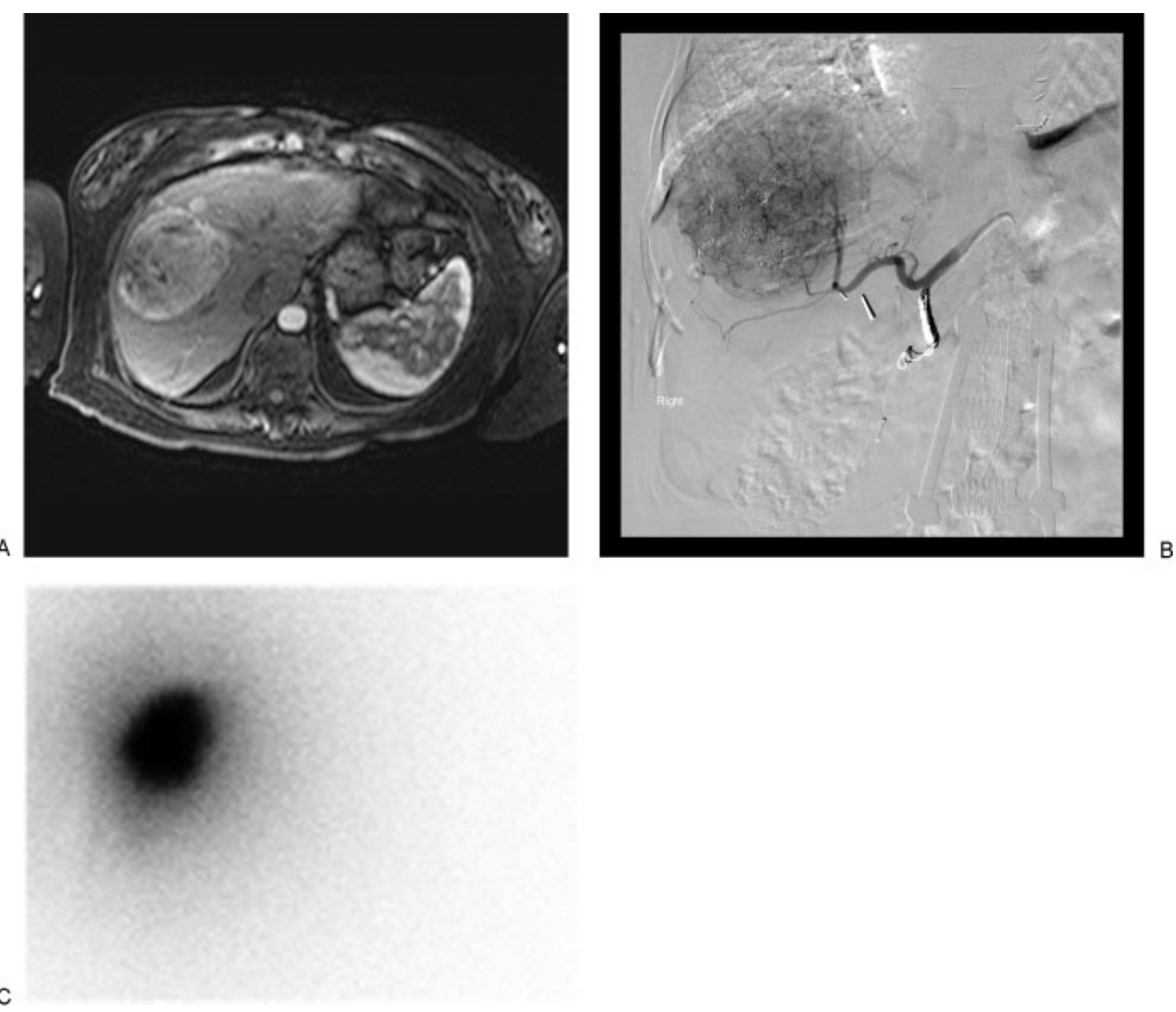

Figure 3 (A) Magnetic resonance imaging (MRI) examination shows large hepatocellular carcinoma in right lobe of liver in 78-year-old woman treated with radioembolization yttrium-90. (B) Hepatic artery angiogram demonstrates a large hypervascular mass in the right lobe, consistent with the findings seen on MRI. Note the gastroduodenal artery was coil embolized on this mapping angiogram before radioembolization therapy. Cholecystectomy surgical clips are noted. (C) Bremsstrahlung scan following radioembolization of right lobe of liver shows radioactivity limited to the right lobe. 
pain, minimal nausea, low-grade fever, and fatigue. It may result in attenuated radiation to adjacent structures, such as the stomach, leading to gastritis. A small pleural effusion may also occur following treatment. After a patient receives radiation treatment, he or she should avoid contact with pregnant women and young children for up to 1 week.

Nontarget administration of microspheres can result in pancreatitis and/or GI tract ulceration. ${ }^{23}$ Another possible complication of radioembolization is radiation hepatitis. The mechanism involves the irradiation of a significant amount of normal liver parenchyma with a presentation similar to veno-occlusive disease. It may cause hepatic fibrosis resulting in portal hypertension. Follow-up is usually with diffusionweighted MRI and, in cases where the tumor was PET avid, a PET scan gives important information about the metabolic activity of the tumor.

Sangro et al studied $24 \mathrm{HCC}$ patients with Child-Pugh A disease who underwent radioembolization with resin microspheres. A reduction in size of lesions in 19 patients was seen, and, using Response Evaluation Criteria in Solid Tumors (RECIST) criteria, $88 \%$ had either partial response or stable disease. No significant postembolization syndrome was noted except jaundice of unknown origin in two cases, and all patients went home within 24 hours of treatment. Two deaths were recorded at 1 and 3 months. At median follow-up of 12.5 months none of the treated patients' disease progressed. ${ }^{24}$ Although initial results and literature have demonstrated safety and effectiveness of radioembolization, its overall efficacy in tumor-free survival when compared with other forms of embolotherapy is yet to be determined. ${ }^{22,25}$

\section{CONCLUSION}

Embolotherapy in the management of $\mathrm{HCC}$ will continue to play an important role; not only in patients with advanced diseased but also as an important bridge to transplant or surgical resection. The safety and shortterm efficacy of theses techniques has been well established; however, looking at the future, its current small survival benefit needs to be improved. The efforts should include development of more potent chemotherapeutic agents, new drugs for specific molecular targeting, and novel therapies such as antiangiogenic agents. The combined use of embolotherapy with other locoregional modalities, such as percutaneous tumor ablation and intratumoral ethanol injection, should be further studied. Improvement in selection criteria for patients and safety profiles of embolizing agents, particularly in employing radioembolization, need to be further elucidated. The embolization protocol that achieves maximal clinical benefit and reduces complications should be tailored to each patient, depending on the patient's amount of tumor and residual liver function. Optimal imaging protocols that demonstrate the efficacy of embolotherapy and provide continued surveillance in a liver at risk for recurrent disease should be established. If we focus on these important parameters, we can significantly improve the survival of patients with HCC.

\section{REFERENCES}

1. Blum HE. Hepatocellular carcinoma: therapy and prevention. World J Gastroenterol 2005;11(47):7391-7400

2. Tanaka Y, Hanada K, Mizokami M, et al. Inaugural Article: A comparison of the molecular clock of hepatitis $\mathrm{C}$ virus in the United States and Japan predicts that hepatocellular carcinoma incidence in the United States will increase over the next two decades. Proc Natl Acad Sci U S A 2002; 99(24):15584-15589

3. Del Pozo AC, López P. Management of hepatocellular carcinoma. Clin Liver Dis 2007;11(2):305-321

4. Befeler AS. Chemoembolization and bland embolization: a critical appraisal. Clin Liver Dis 2005;9(2):287-300 vii

5. Kulik LM. Advancements in hepatocellular carcinoma. Curr Opin Gastroenterol 2007;23(3):268-274

6. Okuda K, Ohtsuki T, Obata H, et al. Natural history of hepatocellular carcinoma and prognosis in relation to treatment. Study of 850 patients. Cancer 1985;56(4):918-928

7. Zhu AX. Development of sorafenib and other molecularly targeted agents in hepatocellular carcinoma. Cancer 2008; 112(2):250-259

8. Covey AM, Maluccio MA, Schubert J, et al. Particle embolization of recurrent hepatocellular carcinoma after hepatectomy. Cancer 2006;106(10):2181-2189

9. Brown KT. Fatal pulmonary complications after arterial embolization with 40-120- micro $m$ tris-acryl gelatin microspheres. J Vasc Interv Radiol 2004;15(2 Pt 1):197-200

10. Izaki K, Sugimoto K, Sugimura K, Hirota S. Transcatheter arterial embolization for advanced tumor thrombus with marked arterioportal or arteriovenous shunt complicating hepatocellular carcinoma. Radiat Med 2004;22(3):155-162

11. Lopez PM, Villanueva A, Llovet JM. Systematic review: evidence-based management of hepatocellular carcinoma-an updated analysis of randomized controlled trials. Aliment Pharmacol Ther 2006;23(11):1535-1547

12. Cammà C, Schepis F, Orlando A, et al. Transarterial chemoembolization for unresectable hepatocellular carcinoma: meta-analysis of randomized controlled trials. Radiology 2002; 224(1):47-54

13. Llovet JM, Real M, Montaña X, et al. Arterial embolisation or chemoembolisation versus symptomatic treatment in patients with unresectable hepatocellular carcinoma: a randomised controlled trial. Lancet 2002;359(9319):1734-1739

14. Lo CM, Ngan H, Tso WK, et al. Randomized controlled trial of transarterial lipiodol chemoembolization for unresectable hepatocellular carcinoma. Hepatology 2002;35(5): 1164-1171

15. Stuart K. Chemoembolization in the management of liver tumors. Oncologist 2003;8(5):425-437

16. Ensminger WD, Gyves JW. Clinical pharmacology of hepatic arterial chemotherapy. Semin Oncol 1983;10(2): 176-182

17. Lewis AL, Gonzalez MV, Lloyd AW, et al. DC bead: in vitro characterization of a drug-delivery device for transarterial 
chemoembolization. J Vasc Interv Radiol 2006;17(2 Pt 1): 335-342

18. Qian J, Truebenbach J, Graepler F, et al. Application of polylactide-co-glycolide-microspheres in the transarterial chemoembolization in an animal model of hepatocellular carcinoma. World J Gastroenterol 2003;9(1):94-98

19. Poon RT, Tso WK, Pang RW, et al. A phase I/II trial of chemoembolization for hepatocellular carcinoma using a novel intra-arterial drug-eluting bead. Clin Gastroenterol Hepatol 2007;5(9):1100-1108

20. Constantin M, Fundueanu G, Bortolotti F, et al. Preparation and characterisation of poly(vinyl alcohol)/cyclodextrin microspheres as matrix for inclusion and separation of drugs. Int J Pharm 2004;285(1-2):87-96

21. Murthy R, Nunez R, Szklaruk J, et al. Yttrium-90 microsphere therapy for hepatic malignancy: devices, indications, technical considerations, and potential complications. Radiographics 2005;25(Suppl 1):S41-S55
22. Kennedy A, Nag S, Salem R, et al. Recommendations for radioembolization of hepatic malignancies using yttrium-90 microsphere brachytherapy: a consensus panel report from the radioembolization brachytherapy oncology consortium. Int J Radiat Oncol Biol Phys 2007;68(1):13-23

23. Kulik LM, Atassi B, van Holsbeeck L, et al. Yttrium-90 microspheres (TheraSphere) treatment of unresectable hepatocellular carcinoma: downstaging to resection, RFA and bridge to transplantation. J Surg Oncol 2006;94(7):572586

24. Sangro B, Bilbao JI, Boan J, et al. Radioembolization using 90Y-resin microspheres for patients with advanced hepatocellular carcinoma. Int J Radiat Oncol Biol Phys 2006; 66(3):792-800

25. Ibrahim SM, Lewandowski RJ, Sato KT, et al. Radioembolization for the treatment of unresectable hepatocellular carcinoma: a clinical review. World J Gastroenterol 2008; 14(11):1664-1669 